\title{
Advanced Encryption Standard Encryption
}

\section{AES Design}

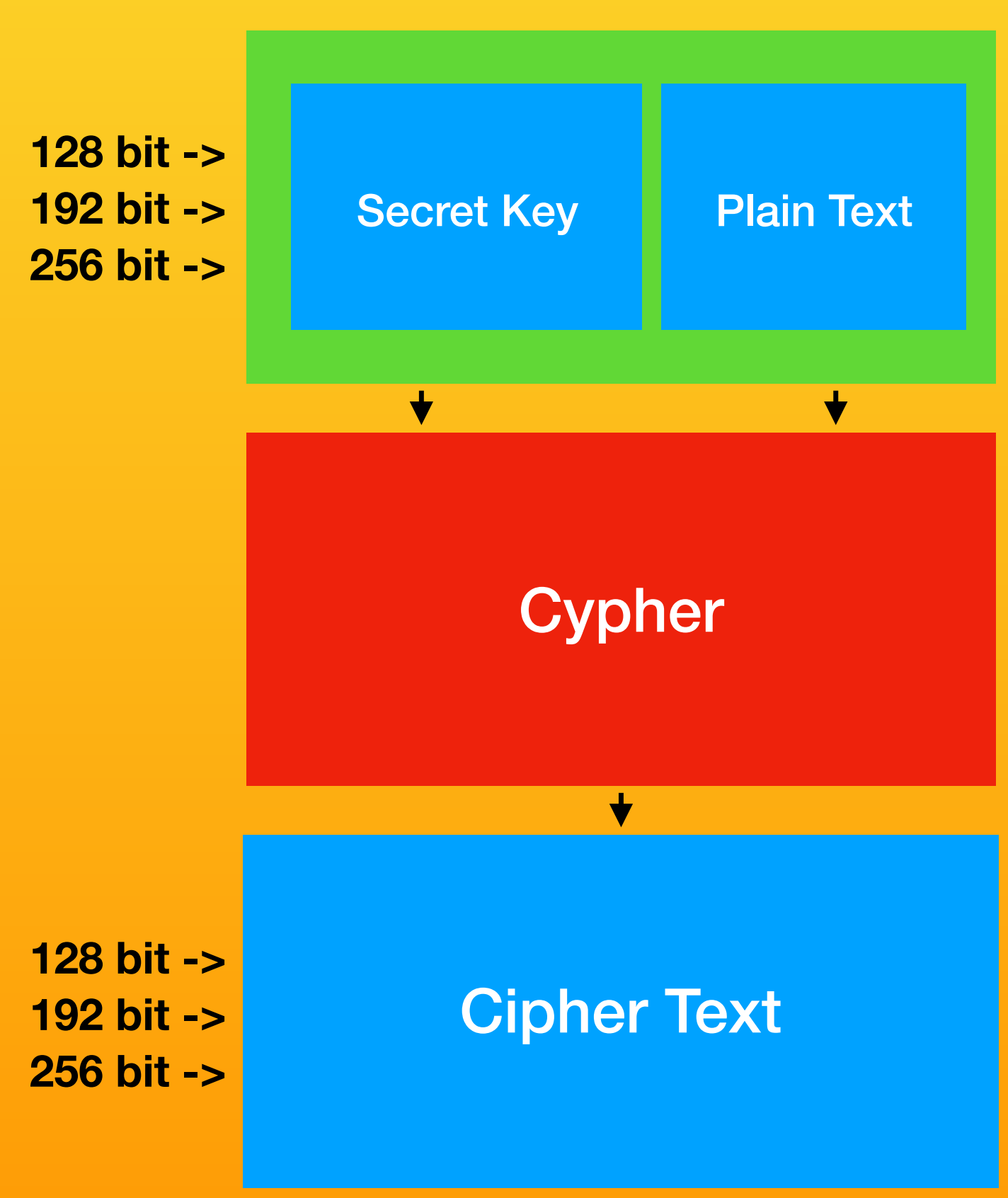

\section{What is AES?}

The Advanced Encryption Standard (AES) is a symmetric block cipher chosen by the U.S. government to protect classified information. AES is implemented in software and hardware throughout the world to encrypt sensitive data. It is essential for government computer security, cybersecurity and electronic data protection.

\section{Features of AES}

Security. Competing algorithms were to be judged on their ability to resist attack -- as compared to other submitted ciphers. Security strength was to be considered the most important factor in the competition.

Cost. Intended to be released on a global, nonexclusive and royalty-free basis, the candidate algorithms were to be evaluated on computational and memory efficiency.

Implementation. Factors to be considered included the algorithm's flexibility, suitability for hardware or software implementation, and overall simplicity. 